\title{
CALIDAD DE LA CARNE DE POTRO GALLEGO DE MONTE EN DIFERENTES EXPLOTACIONES
}

\author{
MEAT QUALITY OF GALICIAN MOUNTAIN FOALS FROM THREE DIFFERENT FARMS
}

Franco, D. ${ }^{1}$, Fernández, M. ${ }^{1}$, Rodríguez, E. ${ }^{1}$, García, L. ${ }^{1}$ y Lorenzo, J.M. ${ }^{1 \star}$

${ }^{1}$ Centro Tecnológico de la Carne. San Cibrao das Viñas. Ourense. España. *jmlorenzo@ceteca.net

\section{Palabras CLAVE ADICIONALES}

Composición. Color. Parámetros de textura.

\section{RESUMEN}

Se estudió la calidad de la carne del músculo Longissimus dorsi (LD) en 51 Potros Gallegos de Monte procedentes de tres explotaciones diferentes. El sistema de explotación afectó al contenido de humedad y proteína e índice de rojo $(p<0,001)$, y también afectó al contenido de Fe-hemo y al índice de amarillo $(p<0,01)$, aunque estas diferencias también pueden ser debidas a la distinta edad de sacrificio de los animales estudiados. En general, la carne fue muy magra ( $<1 \%$ en GIM) con un alto contenido de proteína $(>19,5 \%$ ) y Fe-hemo (1,47 mg/100 g carne) comparado con la carne de ternera. Las muestras también mostraron una alta luminosidad $\left(L^{*}>40\right)$ muy buena capacidad de retención de agua, medida a través de las pérdidas por cocción (<17.4\%) y una terneza inferior a $4 \mathrm{~kg}$, pudiéndose clasificar como muy tiernas.

\section{SUMMARY}

The meat quality of Longissimus dorsi (LD) in a total of 51 Galician Mountain Foals from three diferent production systems was studied. The livestock production system affected significantly moisture and protein content and redness $(p<0.001)$, also affected on heme-Fe content and yellowness $(p<0.01)$, although these differences could be also at differences in slaughter aged of foals . In general, meat from foals of this study showed as a very lean meat ( $<1 \%$ in IMF) with a high protein content $(>19.5 \%)$ and heme-Fe (1.47 $\mathrm{mg} / 100 \mathrm{~g}$ meat) comparable to veal meat. Also, meat samples showed, higher luminosity $\left(L^{*}>40\right)$

Presentado al Congreso SERGA (2010, Asturias).

\author{
AdDitional KEYWORDS \\ Composition. Colour. Texture parameters.
}

very good water holding capacity, measured by cooking losses $(<17.4 \%)$ and tenderness less than $4 \mathrm{~kg}$, so can be classified as a meat very tender.

\section{INTRODUCCIÓN}

La producción mundial de carne de caballo para el consumo en 2007 fue en general de interés marginal (sólo el 0,3\%) en comparación con las de cerdo, vacuno, pollo y pavo (FAOSTAT, 2007). La carne de potro se puede considerar nutricionalmente excelente, con bajo contenido graso, rico en Fehemo, bajo colesterol y un perfil de ácidos grasos con altos niveles de insaturados y vitamina B (Lorenzo et al., 2010).

Caballo Gallego de Monte es una denominación de la población equina explotada en régimen de libertad en los montes de Galicia relacionada directamente con el Caballo de Pura Raza Gallega con un tronco común, el tronco Celta, y puede constituirse como una agrupación racial en fase de estudio que conduzca a un futuro reconocimiento oficial (Fernández et al., 2001). Dada la importancia del censo actual (más de 20000 cabezas), el objetivo del presente trabajo es evaluar las características de la carne del Potro Gallego de Monte en tres explotaciones representativas de diferentes sistemas de producción. 


\section{MATERIAL Y MÉTODOS}

Se han utilizado 51 animales, provenientes de tres explotaciones: sistema extensivo en régimen de libertad (SEL), son animales de gran rusticidad muy adaptados al ambiente donde viven, los montes de la localidad de Sabucedo (Pontevedra, España), con condiciones extremas de alimentación y climatológicas en un sistema de curros, sacrificados a los 212 días; sistema de explotación extensivo (SE), con animales provenientes de la Asociación Monte Cabalar de A Estrada (Pontevedra, España) que consiste en un sistema extensivo pero vallado donde el animal está sometido a un mayor grado de manejo por parte de los dueños (tratamientos veterinarios, control de pesos, etc), sacrificados a los 369 días y, por último, sistema de explotación extensiva con acabado (SEC) con animales del rebaño experimental del Centro de Investigaciones Agrarias de Mabegondo, que han permanecido con sus madres en pastoreo, a excepción de los últimos tres meses que fueron alimentados con heno y pienso ecológico, sacrificados a los 313 días.

Los animales fueron sacrificados en un matadero comercial (A Estrada, Pontevedra, España). Después del sacrificio, las canales fueron pesadas y enfriadas a $4^{\circ} \mathrm{C}$ en cámara refrigerada durante 24 horas. La canal izquierda fue trasladada a la planta piloto del Centro Tecnológico de la Carne donde se extrajo el músculo Longissimus dorsi (LD) entre la $4^{\mathrm{a}}$ y $10^{\mathrm{a}}$ costilla. El LD fue envasado a vacío a una temperatura de $4^{\circ} \mathrm{C}$, en bolsas de poliestireno, durante 4 días.

La medida del pH se realizó usando un pH-metro portátil (Hanna instruments) equipado con electrodo de penetración de $6 \mathrm{~mm}$ de diámetro y una sonda de temperatura. El contenido en humedad, grasa intramuscular y proteína se determinaron empleando las Norma Internacionales ISO R-1442 (ISO, 1978), ISO R-1443 (ISO, 1978) y ISO R-937 (ISO, 1978), respectivamente.

La capacidad de retención de agua de la carne se determinó mediante las pérdidas por goteo y pérdidas por cocción.

El color se determinó con espectrofotómetro portátil MINOLTA serie CR-400 con la ayuda de las coordenadas tricromáticas: $L^{*}$ (luminosidad), $a^{*}$ (índice de rojo) y $b^{*}$ (índice de amarillo). El contenido en Fehemo fue medido según el método de Hornsey (1956) con la siguiente expresión (Merck, 1989):

hematina ( $\mu \mathrm{g}$ hematina/g músculo $)=$ absorbancia $\times 342,44$;

Fe-hemo $(\mathrm{mg} / 100 \mathrm{~g}$ carne $)=($ hematina $\times 8,82) /$ 100.

La dureza fue determinada sobre muestras de carne de $1 \mathrm{x} 1 \mathrm{x} 1 \mathrm{~cm}$, en un texturómetro TA. XTPlus (Stable Micro Systems, Surrey, UK) con célula Warner-Bratzler aplicada al texturómetro y velocidad de ensayo de 3,33 $\mathrm{mm} / \mathrm{s}$, cortando la muestra perpendicularmente a las fibras del músculo.

Los resultados se analizaron mediante ANOVA con el programa SPSS 18.0 considerando el efecto explotación. Se aplicó el test de Duncan cuando la diferencia entre medias era significativa.

\section{RESULTADOSYDISCUSIÓN}

En las tabla I se recogen los valores medios, desviación estándar y el análisis de varianza del efecto de la explotación sobre la composición química de la carne, los parámetros de color, capacidad de retención de agua y los parámetros de textura.

La explotación afectó muy significativamente $(p<0,001)$ al contenido de humedad, proteína e índice de rojo y de manera menos significativa al contenido de Fe-hemo e índice de amarillo ( $<<0,01)$, aunque estas diferencias también pueden ser debidas en parte a la distinta edad de sacrificio de los animales.

El sistema de explotación no afectó de forma significativa al $\mathrm{pH}$. Valores similares fueron obtenidos por Polidori et al. (2008) en carne de burro, Sarriés y Beriain (2005) y 
en carne de potros de las razas Burguete y Halfinger, respectivamente (Lanza et al., 2009). El contenido medio de agua en las muestras de carne de potro del LD (mayor del $75 \%$ ) fue superior al encontrado en la bibliografía (valores próximos al 72\%) para carne equina (Polidori et al., 2008; Juárez et al., 2009; Lanza et al., 2009).

El acabado de los potros durante tres meses aumentó el contenido graso en la carne de los mismos (0,68 y 0,83 vs. 0,92$)$ aunque no fue significativo ( $\mathrm{p}>0,05)$, siendo dichos valores inferiores a los publicados por otros investigadores (Juárez et al., 2009; Lanza et al., 2009). Estas diferencias también pueden ser debidas a las distintas edades de sacrifico, ya que se asume que a medida que el animal envejece va depositando mayor cantidad de grasa. Los bajos niveles de grasa registrados podrían por tanto ser atribuidos no solo a la genética, sino a que se sacrificaron en un periodo muy temprano de crecimiento del animal. El con- tenido de proteína si fue comparable al mostrado por Sarries y Beriain (2005) en carne de potro.

Los sistemas de producción juegan un papel importante en la diferenciación del color de la carne, así, los potros con acabado presentaron valores más altos de $\mathrm{a}^{*} \mathrm{y}$ una menor luminosidad. Los valores de $\mathrm{L}^{*}$ son mucho mayores que los encontrados por Sarries y Berian (2006) $(34,80)$. Estas diferencias podrían atribuirse a la diferente raza de caballo y a la diferencias en la edad de sacrificio. En este sentido hay discrepancia, puesto que Gatellier et al. (2005) no encontraron diferencias en la luminosidad de la carne de ternera atribuibles a la edad, mientras que Dufey, (1996) en Longissimus thoracis de caballos sacrificados a los $30 \mathrm{y}$ 7 meses encontró valores de 33,02 y 37,17, respectivamente, mostrándose los valores de L * más bajos en los animales más viejos.

En el trabajo de Sarries y Berian (2006) el sistema de explotación no afectó a la fuerza

Tabla I. Efecto del sistema de explotación sobre la calidad de la carne de Potro Gallego de Monte. (Effect of livestock production system on meat quality of Galician Mountain Foals).

\begin{tabular}{|c|c|c|c|c|c|c|}
\hline & & & ema de explot & & & \\
\hline & & SEL & SE & SEC & SEM & Sig \\
\hline & Animales & 14 & 17 & 20 & & \\
\hline & $\mathrm{pH}$ & $5,55 \pm 0,12$ & $5,63 \pm 0,05$ & $5,62 \pm 0,12$ & 0,01 & n.s. \\
\hline$\frac{0}{\frac{0}{n}}$ & Humedad \% & $76,40 \pm 1,23^{a}$ & $76,95 \pm 0,81^{a}$ & $75,07 \pm 0,69^{b}$ & 0,17 & 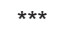 \\
\hline 足 & Grasa intramuscular \% & $0,68 \pm 0,41$ & $0,83 \pm 0,44$ & $0,92 \pm 0,34$ & 0,05 & n.s. \\
\hline ह & Proteína \% & $21,12 \pm 1,17^{a}$ & $19,15 \pm 0,65^{b}$ & $20,46 \pm 0,69^{c}$ & 0,16 & *** \\
\hline & Fe-hemo \% & $1,47 \pm 0,35^{a}$ & $1,64 \pm 0,21^{a b}$ & $1,85 \pm 0,32^{b}$ & 0,04 & ** \\
\hline & Perdidas por cocción & $1,10 \pm 0,46^{a}$ & $0,84 \pm 0,31^{b}$ & $0,95 \pm 0,27^{\mathrm{ab}}$ & 0,04 & * \\
\hline 品 & Perdidas por goteo & $21,43 \pm 5,12$ & $17,39 \pm 4,69$ & $19,45 \pm 3,58$ & 0,64 & n.s. \\
\hline & Luminosidad (L*) & $44,73 \pm 3,24^{a}$ & $43,58 \pm 3,76^{b}$ & $41,90 \pm 1,74^{b}$ & 0,43 & * \\
\hline 흥 & Índice de rojo $\left(a^{\star}\right)$ & $17,07 \pm 1,80^{a}$ & $17,71 \pm 1,84^{\mathrm{a}}$ & $20,49 \pm 2,28^{b}$ & 0,35 & 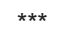 \\
\hline O & Índice de amarillo (b*) & $13.23 \pm 0.82^{a}$ & $11,87 \pm 1,49^{b}$ & $13,44 \pm 1,42^{c}$ & 0,20 & ** \\
\hline & Fuerza de corte $\left(\mathrm{kg} / \mathrm{cm}^{2}\right)$ & $3,84 \pm 1,40$ & $3,30 \pm 0,85$ & $3,15 \pm 0,97$ & 0,15 & n.s \\
\hline $\bar{z}$ & Firmeza al corte & $0,81 \pm 0,31$ & $0,85 \pm 0,22$ & $0,86 \pm 0,25$ & 0,03 & n.s \\
\hline$\Phi$ & Trabajo total de corte & $17,10 \pm 6,21$ & $12,97 \pm 2,77$ & $13,87 \pm 4,82$ & 0,68 & n.s \\
\hline
\end{tabular}

CRA: Capacidad de retencion de agua (\%); ${ }^{* \star} \mathrm{p}<0.001 ;{ }^{* *} \mathrm{p}<0.01 ;{ }^{*} \mathrm{p}<0.05$; SEL: extensivo en régimen de libertad; SE: extensivo; SEC: extensivo con acabado. 


\section{FRANCO, FERNÁNDEZ, RODRÍGUEZ, GARCÍA Y LORENZO}

de corte en filetes madurados durante 4 días. En ganado vacuno es conocido que el sistema de explotación afecta, y mucho, a la terneza de la carne, concretamente Vestergaard et al. (2000), encontraron que la carne de toros alimentados en régimen extensivo, fue más dura que la de animales en régimen intensivo.

\section{BIBLIOGRAFÍA}

Dufey, P.A. 1996. Sensory and physicochemical properties of meat from horses of different age groups. In: Procceding $42^{\text {th }}$ International of Meat Science and Technology. Lillehamer. Norway. pp: 556-557.

Fernández, M., Rivero, G., Alonso, M., Rivero, C.J., Pose, H., Justo, J.R., Adán, S., Díaz, R., Rois, D. y Carril, J.A. 2001. Razas autóctonas de Galicia en peligro de extinción. Servicio de Estudios y Publicaciones de la Consellería de Política Agroalimentaria e Desenvolvemento Rural. Xunta de Galicia. Santiago.

FAOSTAT. 2007. Production- Livestock primary. On line database of the Food and Agriculture Organization of the United Nations. http:// faostat.fao.org (02/05/2010).

Gatellier, P., Mercier, Y., Juin, H. and Renerre, M. 2005. Effect of finishing mode (pasture or mixed diet) on lipid composition, colour stability and lipid oxidation in meat from Charolais cattle. Meat Sci., 69: 175-186.

Honikel K.O. 1997. Reference methods supported by OECD and their use in Mediterranean meat products. Food Chem. 5: 573-582.

Hornsey, H.C. 1956. The colour of cooked cured pork estimation of the nitric-oxide ham pigments. J. Sci. Food Agri., 7: 534-540.

ISO. 1973. Determination of total fat content. ISO 1443:1973 Standard. In: International Standards Meat and Meat Products. International Organization for Standardization. Ginebra. Suiza.

ISO. 1978. Determination of nitrogen content. ISO 937:1978 Standard. In: International Standards Meat and Meat Products. International Organization for Standardization. Ginebra. Suiza.

ISO. 1997. Determination of moisture content. ISO

\section{AGRADECIMIENTOS}

A la Consellería do Medio Rural de la Xunta de Galicia (Proyecto FEADER 200703) por la financiación. A las cooperativas Monte Cabalar y Sabucedo Puxa y al Centro de Investigaciones Agrarias de Mabegondo por las canales suministradas.

1442:1997 Standard. In: International Standards Meat and Meat Products. International Organization for Standardization. Ginebra. Suiza.

Juárez, M., Polvillo, O. Gómez, M.D., Alcalde, M.J., Romero, F. and Varela, M. 2009. Breed effect on carcass and meat quality of foals slaughtered at 24 months of age. Meat Sci., 83: 224-228.

Lorenzo, J.M., Fuciños, C., Purriños, L. and Franco, D. 2010. Intramuscular fatty acid composition of Galician Mountain foals breed. Effect of sex, slaughtered age and livestock production system. Meat Sci., 86: 825-831.

Lanza, M., Landi, C., Scerra, M., Galofaro, V. and Pennisi, P. 2009. Meat quality and intramuscular fatty acid composition of Sanfratellano and Haflinger foals. Meat Sci., 81:142-147.

Merck index. 1989. An encyclopedia of chemicals, drugs and biologicals. $11^{\text {th }}$ ed. Merck and Company. Rahway, NJ. 2240 pp.

Polidori, P., Vincenzetti, S., Cavallucci, C. and Beghelli, D. 2008. Quality of donkey meat and carcass characteristics. Meat Sci., 80: 12221224.

Sarriés, M.V. and Beriain, M.J. 2005. Carcass characteristics and meat quality of male and female foals. Meat Sci., 70: 141-152.

Sarriés, M.V. and Beriain, M.J. 2006. Colour and texture characteristics in meat of male and female foals. Meat Sci., 74: 738-745.

Vestergaard, M., Therkildsen, M., Henckel, P., Jensen, L.R., Andersen, H.R. and Sejrsen, K. 2000. Influence of feeding intensity, grazing and finishing feeding on meat and eating quality of young bulls and the relationship between muscle fibre characteristics, fibre fragmentation and meat tenderness. Meat Sci., 54: 187-195.

Archivos de zootecnia vol. 60, núm. 231, p. 392. 